\title{
Determinação da Altura da Camada Limite Planetária na Floresta Ama- zônica Utilizando um Ceilometer
}

\author{
Determination of Planetary Boundary Layer heights in the Forest Amazon Using a \\ Ceilometer
}

\author{
Rayonil Gomes Carneiroํㅜ, Gilberto Fisch², Thomas Kaufmann ${ }^{1}$ \\ ${ }^{1}$ Centro de Previsão do Tempo e Estudos Climáticos (CPTEC), Instituto Nacional de Pesquisas Espaciais (INPE), \\ São José dos Campos - SP - Brasil. E-mail: rayonilcarneiro@gmail.com; \\ ${ }^{2}$ Instituto de Aeronáutica e Espaço (IAE), São José dos Campos, SP, Brasil
}

\begin{abstract}
Resumo
O presente trabalho teve como objetivo determinar o crescimento da Camada Limite Planetária (CLP), através de medidas realizadas por um instrumento de sensoriamento remoto (ceilometer) instalado na região da Floresta Amazônica. Foram utilizados dados provenientes do Projeto GoAmazon (http://campaign.arm.gov/goamazon2014/) localizada próxima ao município de Manacapuru no Estado do Amazonas. Foram analisados dados da altura da CLP, para 45 dias da estação chuvosa (IOP1) entre fevereiro e março, e 45 dias da estação seca (IOP2) entre setembro e outubro, ambos no ano de 2014. Verificou-se que o Ceilometer apresentou dados condizentes com outros valores já determinados para a região. As alturas máximas registradas foram de $1.245 \mathrm{~m}$ durante o IOP1 e 1.685 $m$ no IOP2. Foi observado que a erosão da CLN foi mais lenta no IOP1. Embora a transição da CLC para CLN foi maior no IOP2.
\end{abstract}

Palavras-chave: Micrometeorologia, base das nuvens, sensoriamento remoto

\begin{abstract}
This study aimed to determine the average maximum height of the planetary boundary layer (PBL) through data obtained by ceilometer in the Amazon rainforest region. Used data used are from the GoAmazon Project located next to the Manacapuru municipality in the state of Amazon. Data obtained by the ceilometer for PBL height to 45 days of being analyzed rainy season (IOP1) and 45 days of drought station (IOP2) both in the year 2014. It was found that the ceilometer presented data consistent with that expected for the region. The maximum recorded were 1.245 (IOP1) and 1.685 (IOP2), it was observed that the erosion of the CLN was slower in IOP1. While the transition from CLC for CLN was longer in IOP2. ), it was observed that the erosion of the CLN was slower in IOP1. While the transition from CLC for CLN was longer in IOP2.
\end{abstract}

Keywords: Micrometeorology, cloud base, remote sensing 


\section{Introdução}

A porção mais baixa da troposfera é denominada de Camada Limite Planetária (CLP), sendo a responsável pelas trocas de energia entre a superfície e para a atmosfera (Wallace e Hobbs, 2006). Ela tem forte influência no clima, bem como também é onde encontra-se grande parte dos seres vivos e das atividades humanas (Barlow, 2014). Conhecer a altura máxima da CLP e seu ciclo diário é importante pois possibilita informações sobre diversas aplicações, tais como estudos sobre controle e gerenciamento da qualidade do ar, dispersão de poluentes, parâmetro de entrada em modelos atmosféricos, estudos sobre forçantes climáticas, entre outros (Garratt, 1992; Neves e Fisch, 2015).

Em função da altura máxima da CLP variar bastante ao longo do ciclo diário e, da sua importância acima mencionada, medições continuas são necessárias para se obter um maior entendimento da CLP na região em estudo. As medições de radiossondagem, outra fonte importante de dados da CLP, ocorrem de forma esporádica (um lançamento a cada 6 horas, durante experimentos de campo ou a cada 12 horas na rede sinótica). Assim, instrumentos que fazem uso da técnica de sensoriamento remoto vêm sendo utilizados para se obter uma maior amostragem do ciclo diário da CLP (Emeis, 2010). Portanto, o objetivo deste trabalho é demostrar a utilização de um instrumento de medição da altura da CLP (Ceilometer) para obtenção das alturas da CLP na região da Floresta Amazônica.

\section{Metodologia}

Os dados utilizados neste trabalho foram obtidos durante a campanha do Projeto GoAmazon (http://campaign.arm.gov/goamaz on2014/), realizada na cidade de Manacapuru $\left(03^{\circ} 17^{\prime} 59^{\prime \prime} \mathrm{S}\right.$; $60^{\circ}$ 37' 14" O; alt. $60 \mathrm{~m}$ ) no estado do Amazonas. A cobertura florestal da região é considerada como floresta primária densa de terrafirme.

No local foi instalado um Ceilometer modelo CL31 da Vaisala Inc. (Finlândia) (Figura 1). Os Ceilometers são instrumentos de sensoriamento remoto do tipo LIDAR, que registram a intensidade do retroespalhamento óptico no infravermelho próximo, por meio da emissão de um pulso de luz na vertical, obtendo uma estimativa da base das nuvens, que corresponde aproximadamente a altura da CLP. Este instrumento tem como vantagem em relação a radiossonda sua alta taxa de amostragem e sua maior resolução vertical, porém possui um alto custo, além de apresentar algumas ambíguas. interpretações

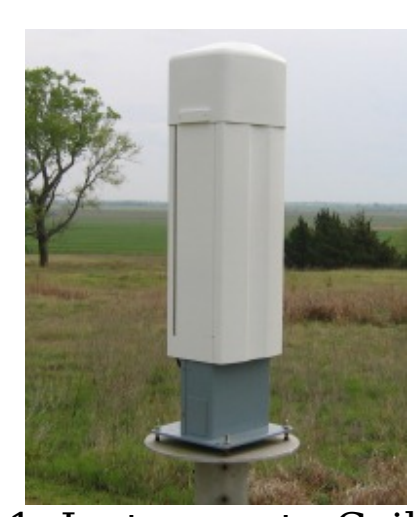

Figura 1. Instrumento Ceilometer. 
Para verificar a precipitação durante os períodos de estudo, foi utilizado dados de um Disdrometro modelo Parsivel $^{2}$ da OTT Hydromet $\mathrm{GmbH}$ (Alemanha) (Figura 2). O qual através de seus dados podem ser calculado o tipo, quantidade e intensidade da precipitação. Assim foi obtida a precipitação em milímetro para cada minuto e realizado o acumulado em cada 10 minutos.

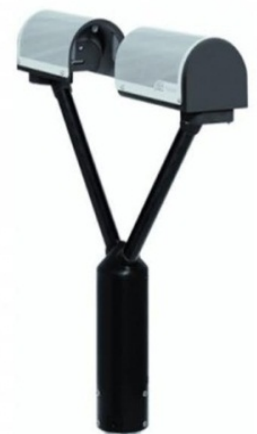

Figura 2. Instrumento Laser Disdrometro.

Foram realizadas análise para 45 dias da estação chuvosa (IOP1), entre 15 de fevereiro e 31 de março de 2014, e para 45 dias da estação seca (IOP2), entre 01 de setembro a 15 de outubro de 2014.

\section{Resultados e Discussões}

As alturas da CLP para o IOP1 estão apresentadas na Figura 3A. Durante o período entre 00 e $05 \mathrm{HL}$, ocorre a fase estável da CLP, denominada de Camada Limite Noturna (CLN). Esta apresentou uma altura média bastante estável, variando de 217 (02 HL) a 268 (04 HL) m, sendo que a pequena variação com a altura está associada a turbulência mecânica intermitente (Santos et al., 2007).
Os dados apresentaram neste intervalo tem um baixo desvio padrão $(\sigma)$ da ordem de $20 \mathrm{~m} \mathrm{e}$, coeficiente variância $(\mathrm{CV} \mathrm{CV})$ de 8\% (Tabela 1). A partir das 06 até 09 HL ocorre o período de erosão da CLN, onde a estabilidade atmosférica presente começa a ser destruída e, ocorre o início da formação de uma camada instável próxima à superfície (Angevine, 2008). Neste intervalo de tempo observa-se uma elevação da altura da CLP, com taxa de crescimento de, entre $80 \mathrm{~m} \mathrm{~h}^{-1}$ (entre 06 e $08 \mathrm{HL}$ ) e $150 \mathrm{~m} \mathrm{~h}^{-1}$ (entre 08 e $09 \mathrm{HL}$ ). Nesta fase verifica-se um aumento significativo do $\sigma(119 \mathrm{~m})$ e da $C V$ CV (29\%). Por se tratar de uma fase de transição, pode ocorrer um aumento gradativo da convecção (Beare, 2008). Após a CLN ser completamente erodida, começa a se estabelecer a Camada Limite Convectiva (CLC). No intervalo das 09 às $12 \mathrm{HL}$, esta encontra-se em crescimento e considera-se como uma CLC rasa. Em função do aquecimento da superfície devido ao aumento de radiação solar global incidente (ciclo diário da radiação), observa-se um aumento significativo da altura da CLP, com uma taxa de crescimento de aproximadamente $204 \mathrm{~m} \mathrm{~h}^{-1}$ entre 09 às $12 \mathrm{HL}$, quando a CLC torna-se bem estabelecida. Os resultados encontrados nesta fase apresentaram a maior variabilidade de toda a IOP1, com valores de até $255 \mathrm{~m}$ (Tabela 1). Este fato está associado com a formação de chuvas no local (Figura 4A). Na fase em que a CLC já está bem estabelecida (12 à $17 \mathrm{HL})$, neste momento a 
Tabela 1. Desvio Padrão $(\sigma \sigma)$ e Coeficiente de Variação (\%) e a Taxa média de Crescimento $\left(\mathrm{m} \mathrm{h}^{-1}\right)$ dos dados obtidos pelo Ceilometer para os IOP1 e IOP2.

\begin{tabular}{|c|c|c|c|c|c|}
\hline \multicolumn{6}{|c|}{ IOP1 } \\
\hline |Horário & $\begin{array}{c}00-05 \\
\text { HL }\end{array}$ & $\begin{array}{c}06-09 \\
\text { HL }\end{array}$ & $\begin{array}{c}09-12 \\
\text { HL }\end{array}$ & $\begin{array}{c}12-17 \\
\text { HL }\end{array}$ & $\begin{array}{c}17-19 \\
\text { HL }\end{array}$ \\
\hline $\boldsymbol{\sigma}$ & 20 & 119 & 255 & 174 & 150 \\
\hline $\mathrm{CV}$ & 8 & 29 & 27 & 18 & 30 \\
\hline $\begin{array}{c}\text { Taxa de } \\
\text { Crescime } \\
\text { nto }\end{array}$ & -4 & 91 & 204 & -106 & -147 \\
\hline \multicolumn{6}{|c|}{ IOP2 } \\
\hline |Horário & $\begin{array}{c}00-05 \\
\text { HL }\end{array}$ & $\begin{array}{c}06-09 \\
\text { HL }\end{array}$ & $\begin{array}{c}09-12 \\
\text { HL }\end{array}$ & $\begin{array}{c}12-17 \\
\text { HL }\end{array}$ & $\begin{array}{c}17-19 \\
\text { HL }\end{array}$ \\
\hline $\boldsymbol{\sigma}$ & 14 & 314 & 222 & 217 & 256 \\
\hline $\mathrm{CV}$ & 7 & 58 & 16 & 15 & 36 \\
\hline $\begin{array}{c}\text { Taxa de } \\
\text { Crescime } \\
\text { nto } \\
\end{array}$ & 5,7 & 212 & 295 & -120 & -341 \\
\hline
\end{tabular}

camada de mistura praticamente domina toda a CLC, foi verificado pouca variação da altura entre os horários e, registrada a altura média máxima da IOP1 de, aproximadamente, $1.245 \mathrm{~m}$ às 12 HL. Logo após este máximo, a CLC começa a demonstrar uma redução de $90 \mathrm{~m} \mathrm{~h}^{-1}$ até às $15 \mathrm{HL}$. Já entre 15 e 17 HL nota-se uma redução mais acentuada de 200 $\mathrm{m} \mathrm{h}^{-1}$, o que está relacionado à redução da convecção presente, que ocorre neste horário por ser o período chuvoso e, consequentemente, há mais nebulosidade, como pode ser verificado na Figura 4A. A precipitação durante o IOP1 apresentou um total acumulado de $720 \mathrm{~mm}$, com as maiores médias horárias de precipitação localizada no período entre 11 e 16 HL (Figura 4C).

A partir das $17 \mathrm{HL}$, iniciase a formação da CLN, a partir da superfície. Até às $19 \mathrm{HL}$, esta ainda não está tão bem estabelecida (entre 00 e $05 \mathrm{HL}$ ), foi de aproximadamente $200 \mathrm{~m}$, semelhante aos valores obtidos na IOP1. Porém, de forma diferente, as alturas apresentaram pouca oscilação: os resultados de $\sigma$ e de $C V C V$ apresentaram valores $(14 \mathrm{~m}$ e $7 \%$, respectivamente), assim como no caso do IOP1. Na erosão da CLN (06 e $09 \mathrm{HL}$ ), verifica-se uma maior taxa de crescimento da CLP, de $80 \mathrm{~m} \mathrm{~h}^{-1}$ (entre 06 e 07 $\mathrm{HL}$ ), de $200 \mathrm{~m} \mathrm{~h}^{-1}$ (entre 07 e 08 $\mathrm{HL}) \mathrm{e}$, atingindo uma maior taxa de crescimento entre 08 e $09 \mathrm{HL}$, de aproximadamente $570 \mathrm{~m} \mathrm{~h}^{-1}$. Este elevação mais acentuada deve-se a maior incidência de radiação solar na estação seca, resultando que os fluxos de calor sensível tornarem-se positivo mais cedo (Fedorovich et al., 2004). Consequentemente, este intervalo também mostrou um $\sigma$ e o CV CV mais elevados (314 e $58 \%$, respectivamente). 
A)

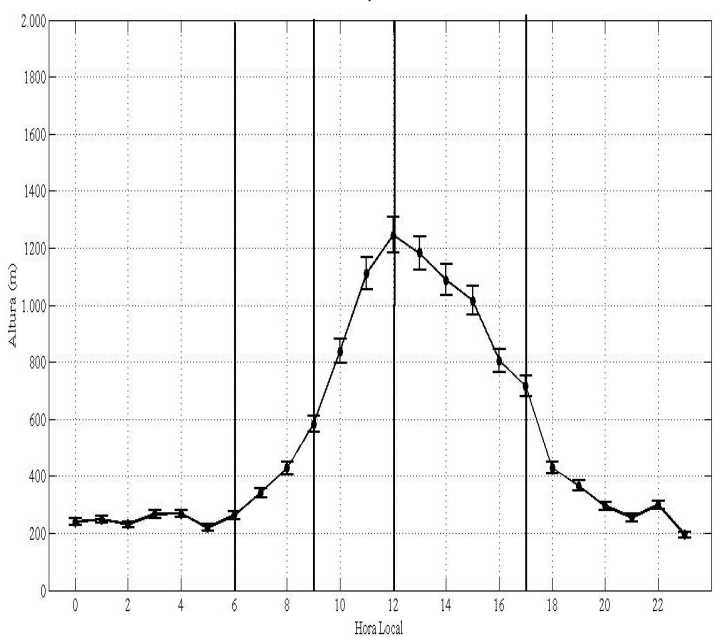

B)

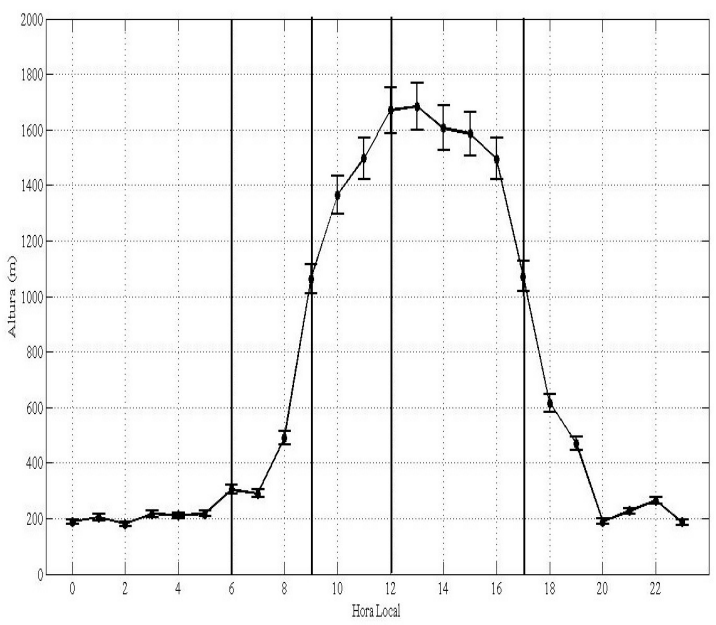

Figura 3. Ciclo médio diário da altura da CLP para a IOP1 (A) e IOP2 (B).

A)

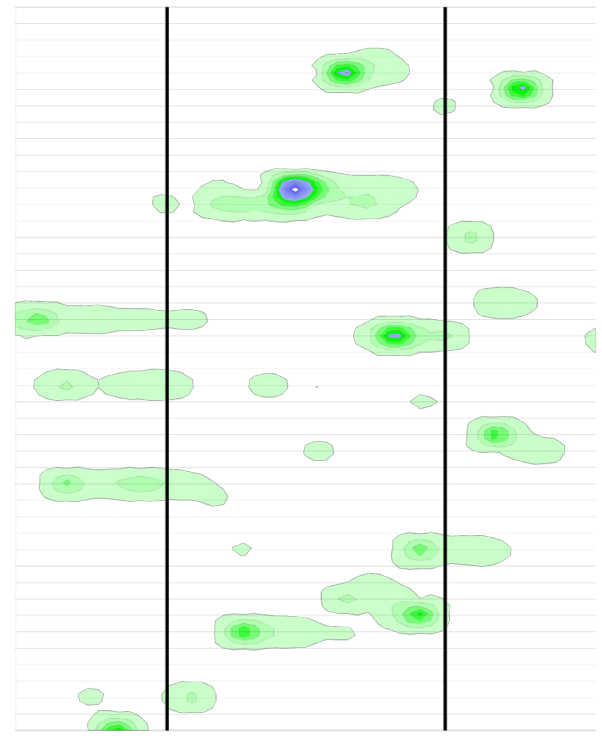

C)

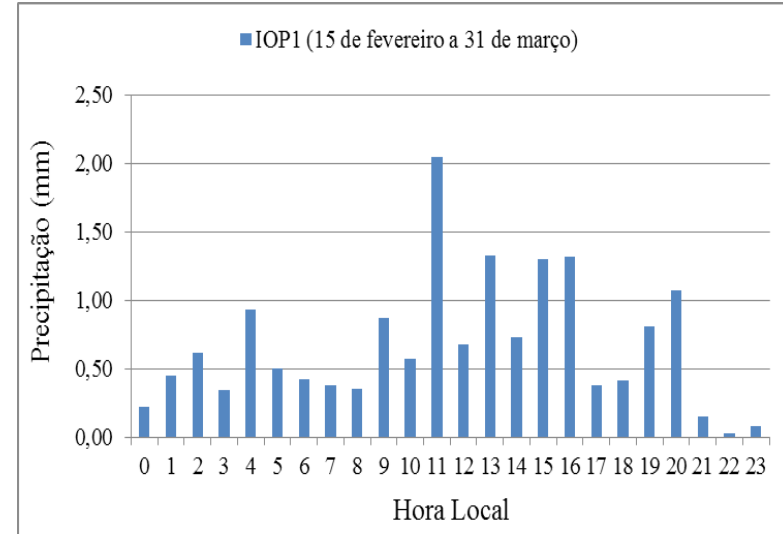

B)

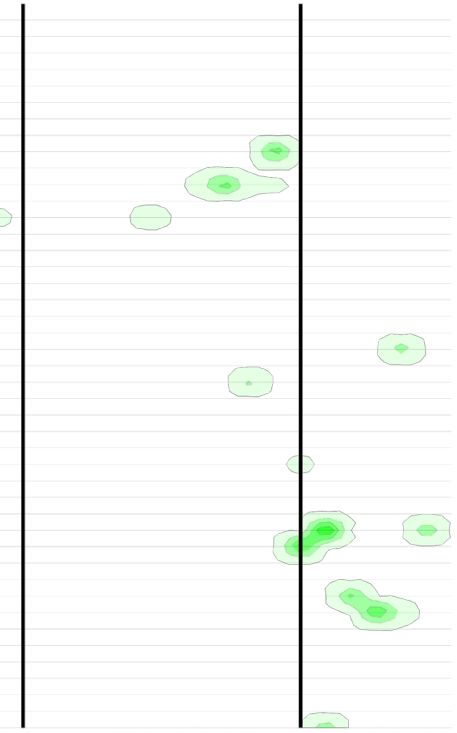

D)

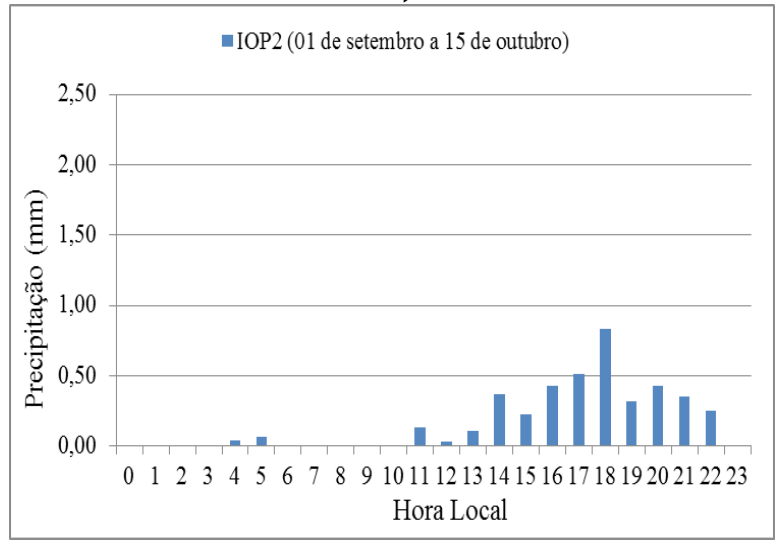

Figura 4. Variação espaço temporal da precipitação para a os períodos IOP1 (A) e IOP2 (B) e média horária da precipitação no IOP1 (C) e IOP2 (D). 
A fase crescimento da CLC (entre 09 e $12 \mathrm{HL}$ ) ocorre mais rapidamente na IOP2, estando completamente estabelecida às $10 \mathrm{HL}$, com altura de $1.364 \mathrm{~m} \mathrm{e}$, uma taxa de crescimento de aproximadamente $150 \mathrm{~m} \mathrm{~h}^{-1}$ a partir deste horário. Neste período de crescimento da CLC, os resultados do $\sigma$ e o do $C V C V$ estiveram semelhantes a da IOP1. Quando a CLC se encontra completamente estabelecida na IOP2, verificou-se uma maior profundidade da CLP: por exemplo, às $13 \mathrm{HL}$, a extensão da CLP é de $1.684 \mathrm{~m}$. Este resultado está de acordo com as análises de Fisch et al. (2004) para a região de Rondônia no sudoeste da Amazônia, e também para Shaw (2005) que afirma que, independente da época do ano e do local, a CLC obtém sua maior profundidade à tarde, devido receber maior influência da turbulência térmica. A CLC fica bem estabelecida e com pouca oscilação até às 16 HL (praticamente estacionária), quando é possível observar uma leve redução, devido ao início do cessar da turbulência térmica. As analises estatísticas nesta fase estiveram em consonância com o mesmo período da estação chuvosa. Entretanto a CLP apresentou profundidade muito mais acentuada neste período no IOP2 em relação ao IOP1.

No intervalo considerado como formação da CLN, diferente da IOP1, às $17 \mathrm{HL}$ ainda existe uma convecção elevada, acarretando que a CLN demore mais a se estabelecer. Isso ocorre, pois a precipitação na IOP2, diferentemente da observada na IOP1, foi bastante escassa
(Figura 4B), com um acumulado de $185 \mathrm{~mm}$ e, com as concentrações médias horárias estando localizadas entre 16 e 22 HL (Figura 4D). Após este intervalo, verificou-se uma forte redução em torno de $450 \mathrm{~m} \mathrm{~h}^{-1}$ (entre 17 e $18 \mathrm{HL}$ ) e ainda com uma altura elevada de $600 \mathrm{~m}$. Notou-se também, que às $19 \mathrm{HL}$ a CLN ainda não se estabeleceu, ao contrário da IOP1, vindo a se estabelecer apenas às $20 \mathrm{HL}$. Em detrimento a isto, os valores de $\sigma$ e o $C V C V$ foram mais elevados.

\section{Comentários finais}

O Ceilometer apresentou resultados satisfatórios do ciclo diário da altura média da CLP, tanto durante o IOP1 como para o IOP2. Na época seca (IOP2), a CLP mostrou-se melhor estabelecida na fase convectiva, atingindo uma maior profundidade máxima em relação a IOP1. Para o período do IOP1, devido a formação de nebulosidade e chuvas, o ciclo diário da CLP não fica muito claro, principalmente no período da tarde.

Os resultados mostraram que a erosão da CLN ocorreu de forma mais lenta na IOP1, enquanto que na IOP2 esta ocorreu de forma mais acentuada, devido a maior convecção precedente e maior energia para sua destruição.

\section{Referências}

Angevine, W. Transitional, entraining, cloudy, and coastal boundary layers.

Acta

Geophysica. v. 56, n. 1, p. $2-$ 20. 2008. 
Barlow, J. F. Progress in observing and modelling the urban boundary layer. Urban Climate. v. 10, p. $216-240$. 2014.

Beare, R. J. The role of shear in the morning transition boundary layer. Boundary Layer Meteorology. v. 129, p. 395 - 410. 2008.

Emeis, S. 2010. Measurement methods in atmospheric sciences, In situ and remote. $1 \stackrel{\mathrm{a} e d .}{ }$ Gebrüder Borntraeger. $257 \mathrm{p}$.

Fedorovich, E.; Conzemius, R.; Mironov, D. Convective entrainment into a shear-free, linearly stratified atmosphere: bulk models reevaluated through large-eddy simulations. Journal of Atmospheric. Sciences. v. 61, p. 281-295. 2004.

Fisch, G.; Tota, J.; Machado, L. A. T.; Silva Dias, M. A. F.; Lyra, R. F. da F.; Nobre, C. A.; Dolmans, A. J.; Gash, J. H. C. The convective boundary layer over pasture and forest in Amazonia.

\section{Theoretical and Applied} Climatology. v 78, p. 47 - 59. 2004.

Garratt, J. R. 1992. The Atmospheric Boundary Layer. Cambridge University Press. $316 \mathrm{p}$.

Neves, T. T. A. T.; Fisch, G. The Daily Cycle of the Atmospheric Boundary Layer Heights over Pasture Site in Amazonia American. Journal of Environmental Engineering. v. 5, p. $39-44.2015$.

Santos, R. M. N.; Fisch, G.; Dolman, A. J.; Waterloo, M. Modelagem da camada limite noturna (CLN) durante a época úmida na Amazônia, sob diferentes condições de desenvolvimento. Revista Brasileira de Meteorologia. v. 22, p. 387-407, 2007.

Shaw, W.; Doran, J.; Coulter, R. Boundary-layer evolution over Phoenix, Arizona and the premature mixing of pollutants in the early morning. Atmospheric Environment. v. 39, n. 4, p. 773-786, Fev. 2005.

Wallace, J. M.; Hobbs, P. V. 2006. Atmospheric Science - An Introductory Survey. $\quad 2^{\mathrm{a}} \mathrm{ed}$. Academic Press. 505p. 